\title{
WEAK AND STRONG CONVERGENCE THEOREMS FOR AN ASYMPTOTICALLY $k$-STRICT PSEUDO-CONTRACTION AND A MIXED EQUILIBRIUM PROBLEM
}

\author{
Yonghong Yao, Haiyun Zhou, and Yeong-Cheng Liou
}

\begin{abstract}
We introduce two iterative algorithms for finding a common element of the set of fixed points of an asymptotically $k$-strict pseudocontraction and the set of solutions of a mixed equilibrium problem in a Hilbert space. We obtain some weak and strong convergence theorems by using the proposed iterative algorithms. Our results extend and improve the corresponding results of Tada and Takahashi [16] and Kim and Xu $[8,9]$.
\end{abstract}

\section{Introduction}

Let $H$ be a real Hilbert space and let $C$ be a nonempty closed convex subset of $H$. Let $\varphi: C \rightarrow \mathbb{R}$ be a real valued function and $\Theta: C \times C \rightarrow \mathbb{R}$ be an equilibrium bifunction, i.e., $\Theta(u, u)=0$ for each $u \in C$. Now we concern the following mixed equilibrium problem (MEP) which is to find $x^{*} \in C$ such that

$$
\Theta\left(x^{*}, y\right)+\varphi(y)-\varphi\left(x^{*}\right) \geq 0, \quad \forall y \in C .
$$

In particular, if $\varphi \equiv 0$, this problem reduces to the equilibrium problem (EP), which is to find $x^{*} \in C$ such that

$$
\Theta\left(x^{*}, y\right) \geq 0, \quad \forall y \in C .
$$

Denote the set of solutions of (MEP) by $\Omega$ and the set of solutions of (EP) by $\Gamma$. The mixed equilibrium problems include fixed point problems, optimization problems, variational inequality problems, Nash equilibrium problems and the equilibrium problems as special cases; see, e.g., [1, 3, 4, 10, 22]. Some methods have been proposed to solve the equilibrium problems and the mixed equilibrium problems, see, e.g., [2, 5, 6, 7, 10, 12, 14, 15, 16, 17, 18, 19, 20, 21].

Received August 25, 2007.

2000 Mathematics Subject Classification. 49J30, 47H10, 47H17, 49M05, 90C25, 90C99.

Key words and phrases. mixed equilibrium problems, fixed point problems, iterative algorithm, asymptotically $k$-strict pseudo-contraction, Hilbert space.

The first two authors were supported by National Natural Science Foundation of China Grant 10771050 and the third author was supported by the grant NSC 97-2221-E-230-017. 
On the other hand, recently, $\mathrm{Kim}$ and $\mathrm{Xu}[8,9]$ introduced some iterative methods for solving fixed point problems of asymptotically nonexpansive mappings and asymptotically $k$-strict pseudo-contractions, respectively. The corresponding iterative algorithms are as follows. The first one introduced in [8] is:

$$
\left\{\begin{array}{l}
x_{0} \in C \text { chosen arbitrarily, } \\
y_{n}=\alpha_{n} x_{n}+\left(1-\alpha_{n}\right) T^{n} x_{n} \\
C_{n}=\left\{z \in C:\left\|y_{n}-z\right\|^{2} \leq\left\|x_{n}-z\right\|^{2}+\theta_{n}\right\} \\
Q_{n}=\left\{z \in C:\left\langle x_{n}-z, x_{0}-x_{n}\right\rangle \geq 0\right\} \\
x_{n+1}=P_{C_{n} \cap Q_{n}} x_{0}
\end{array}\right.
$$

where $T: C \rightarrow C$ is an asymptotically nonexpansive mapping and $\theta_{n}=(1-$ $\left.\alpha_{n}\right)\left(k_{n}^{2}-1\right)(\operatorname{diam} \mathrm{C})^{2} \rightarrow 0$ as $n \rightarrow \infty$. And the second one introduced in [9] is:

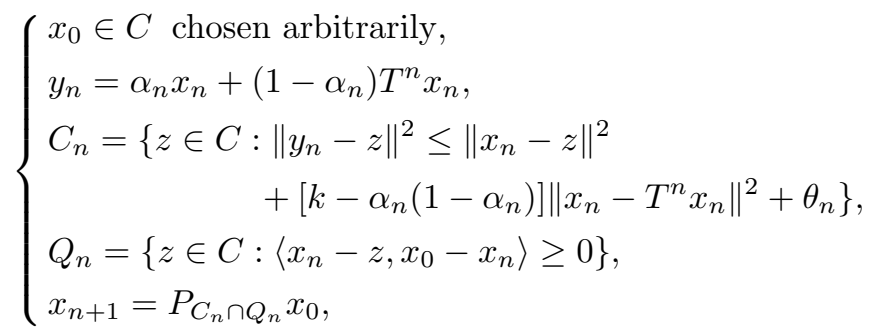

where $T: C \rightarrow C$ is an asymptotically $k$-strict pseudo-contraction and $\theta_{n}=$ $\Delta_{n}^{2}\left(1-\alpha_{n}\right) \gamma_{n} \rightarrow 0(n \rightarrow \infty), \Delta_{n}=\sup \left\{\left\|x_{n}-z\right\|: z \in F(T)\right\}<\infty$. Subsequently, Kim and Xu proved that the iterative algorithm (KX1) and (KX2) are strongly convergent. For more details, see [8, 9]. However, we note that the $(n+1)$ th iterate $x_{n+1}$ is defined as the projection of the initial guess $x_{0}$ onto the intersection of two closed convex subsets $C_{n}$ and $Q_{n}$. Therefore, an interesting problem is how to construct appropriately $C_{n}$ and $Q_{n}$ such that the computations become easier.

Motivated by the above works, in this paper we introduce two iterative algorithms for finding a common element of the set of fixed points of an asymptotically $k$-strict pseudo-contraction and the set of solutions of a mixed equilibrium problem in a Hilbert space. We obtain some weak and strong convergence theorems by using the proposed iterative algorithms. Our results extend and improve the corresponding results of Tada and Takahashi [16], Kim and Xu $[8,9]$.

\section{Preliminaries}

Let $H$ be a real Hilbert space with inner product $\langle\cdot, \cdot\rangle$ and norm $\|\cdot\|$. Let $C$ be a nonempty closed convex subset of $H$. Then, for any $x \in H$, there exists a unique nearest point in $C$, denoted by $P_{C}(x)$, such that

$$
\left\|x-P_{C}(x)\right\| \leq\|x-y\|
$$


for all $y \in C$. Such a $P_{C}$ is called the metric projection of $H$ onto $C$. We know that $P_{C}$ is nonexpansive. Further, for $x \in H$ and $x^{*} \in C$,

$$
x^{*}=P_{C}(x) \Leftrightarrow\left\langle x-x^{*}, x^{*}-y\right\rangle \geq 0 \text { for all } y \in C .
$$

Recall that a mapping $T: C \rightarrow C$ is said to be an asymptotically $k$-strict pseudo-contraction if, there exists a constant $k \in[0,1)$ satisfying

$$
\left\|T^{n} x-T^{n} y\right\|^{2} \leq\left(1+\gamma_{n}\right)\|x-y\|^{2}+k\left\|\left(I-T^{n}\right) x-\left(I-T^{n}\right) y\right\|^{2}
$$

for all $x, y \in C$ and all integers $n \geq 1$, where $\gamma_{n} \geq 0$ for all $n$ and such that $\gamma_{n} \rightarrow 0$ as $n \rightarrow \infty$. Note that if $k=0$, then $T$ is an asymptotically nonexpansive mapping, that is, there exists a sequence $\left\{\gamma_{n}\right\}$ of nonnegative numbers with $\gamma_{n} \rightarrow 0$ such that

$$
\left\|T^{n} x-T^{n} y\right\| \leq\left(1+\gamma_{n}\right)\|x-y\|^{2}
$$

for all $x, y \in C$ and all integers $n \geq 1$.

In the sequel, we will use $F(T)$ to denote the set of fixed points of $T$.

For given sequence $\left\{x_{n}\right\} \subset C$, let $\omega_{w}\left(x_{n}\right)=\left\{x: \exists x_{n_{j}} \rightarrow x\right.$ weakly $\}$ denote the weak $\omega$-limit set of $\left\{x_{n}\right\}$.

In this paper, for solving the mixed equilibrium problems for an equilibrium bifunction $\Theta: C \times C \rightarrow \mathbb{R}$, we assume that $\Theta$ satisfies the following conditions:

(H1) $\Theta$ is monotone, i.e., $\Theta(x, y)+\Theta(y, x) \leq 0$ for all $x, y \in C$;

(H2) for each fixed $y \in C, x \mapsto \Theta(x, y)$ is concave and upper semicontinuous;

(H3) for each $x \in C, y \mapsto \Theta(x, y)$ is convex.

A mapping $\eta: C \times C \rightarrow H$ is called Lipschitz continuous, if there exists a constant $\lambda>0$ such that

$$
\|\eta(x, y)\| \leq \lambda\|x-y\|, \quad \forall x, y \in C .
$$

A differentiable function $K: C \rightarrow \mathbb{R}$ on a convex set $C$ is called:

(i) $\eta$-convex if

$$
K(y)-K(x) \geq\left\langle K^{\prime}(x), \eta(y, x)\right\rangle, \quad \forall x, y \in C,
$$

where $K^{\prime}$ is the Frechet derivative of $K$ at $x$;

(ii) $\eta$-strongly convex if there exists a constant $\sigma>0$ such that

$$
K(y)-K(x)-\left\langle K^{\prime}(x), \eta(y, x)\right\rangle \geq(\sigma / 2)\|x-y\|^{2}, \quad \forall x, y \in C .
$$

Let $C$ be a nonempty closed convex subset of a real Hilbert space $H, \varphi: C \rightarrow$ $\mathbb{R}$ be real-valued function and $\Theta: C \times C \rightarrow \mathbb{R}$ be an equilibrium bifunction. Let $r$ be a positive number. For a given point $x \in C$, the auxiliary problem for (MEP) consists of finding $y \in C$ such that

$$
\Theta(y, z)+\varphi(z)-\varphi(y)+\frac{1}{r}\left\langle K^{\prime}(y)-K^{\prime}(x), \eta(z, y)\right\rangle \geq 0, \quad \forall z \in C .
$$


Let $S_{r}: C \rightarrow C$ be the mapping such that for each $x \in C, S_{r}(x)$ is the solution set of the auxiliary problem, i.e., $\forall x \in C$,

$$
S_{r}(x)=\left\{y \in C: \Theta(y, z)+\varphi(y)+\frac{1}{r}\left\langle K^{\prime}(y)-K^{\prime}(x), \eta(z, y)\right\rangle \geq 0, \forall z \in C\right\} .
$$

We need the following important and interesting result for proving our main results.

Lemma 2.1 ([21]). Let $C$ be a nonempty closed convex subset of a real Hilbert space $H$ and let $\varphi: C \rightarrow \mathbb{R}$ be a lower semicontinuous and convex functional. Let $\Theta: C \times C \rightarrow \mathbb{R}$ be an equilibrium bifunction satisfying conditions (H1)-(H3). Assume that

(i) $\eta: C \times C \rightarrow H$ is Lipschitz continuous with constant $\lambda>0$ such that

(a) $\eta(x, y)+\eta(y, x)=0, \forall x, y \in C$,

(b) $\eta(\cdot, \cdot)$ is affine in the first variable,

(c) for each fixed $y \in C, x \mapsto \eta(y, x)$ is sequentially continuous from the weak topology to the weak topology;

(ii) $K: C \rightarrow \mathbb{R}$ is $\eta$-strongly convex with constant $\sigma>0$ and its derivative $K^{\prime}$ is sequentially continuous from the weak topology to the strong topology;

(iii) for each $x \in C$, there exist a bounded subset $D_{x} \subset C$ and $z_{x} \in C$ such that for any $y \in C \backslash D_{x}$,

$$
\Theta\left(y, z_{x}\right)+\varphi\left(z_{x}\right)-\varphi(y)+\frac{1}{r}\left\langle K^{\prime}(y)-K^{\prime}(x), \eta\left(z_{x}, y\right)\right\rangle<0 .
$$

Then there hold the following:

(I) $S_{r}$ is single-valued;

(II) $S_{r}$ is nonexpansive if $K^{\prime}$ is Lipschitz continuous with constant $\nu>0$ such that $\sigma \geq \lambda \nu$ and

$\left\langle K^{\prime}\left(x_{1}\right)-K^{\prime}\left(x_{2}\right), \eta\left(u_{1}, u_{2}\right)\right\rangle \geq\left\langle K^{\prime}\left(u_{1}\right)-K^{\prime}\left(u_{2}\right), \eta\left(u_{1}, u_{2}\right)\right\rangle, \quad \forall\left(x_{1}, x_{2}\right) \in C \times C$ where $u_{i}=S_{r}\left(x_{i}\right)$ for $i=1,2$;

(III) $F\left(S_{r}\right)=\Omega$;

(IV) $\Omega$ is closed and convex.

We also need the following lemmas.

Lemma 2.2. Let $H$ be a real Hilbert space. There hold the following wellknown identities:

(i) $\|x-y\|^{2}=\|x\|^{2}-2\langle x, y\rangle+\|y\|^{2} \forall x, y \in H$.

(ii) $\|t x+(1-t) y\|^{2}=t\|x\|^{2}+(1-t)\|y\|^{2}-t(1-t)\|x-y\|^{2} \forall t \in[0,1], \forall x, y \in$ $H$.

Lemma $2.3([9])$. Assume $C$ is a closed convex subset of a real Hilbert space $H$ and let $T: C \rightarrow C$ be an asymptotically $k$-strict pseudo-contraction. Then the following conclusions hold: 
(i) for each $n \geq 1, T^{n}$ satisfies the Lipschitz condition:

$$
\left\|T^{n} x-T^{n} y\right\| \leq L_{n}\|x-y\| \forall x, y \in C,
$$

where $L_{n}=\frac{k+\sqrt{1+\gamma_{n}(1-k)}}{1-k}$;

(ii) the mapping $I-T$ is demiclosed at zero, that is, if $\left\{x_{n}\right\}$ is a sequence in $C$ such that $x_{n} \rightarrow x^{*}$ weakly and $(I-T) x_{n} \rightarrow 0$ strongly, then $(I-T) x^{*}=0$;

(iii) the fixed point set $F(T)$ of $T$ is closed and convex so that the projection $P_{F(T)}$ is well-defined.

Lemma 2.4 ([11]). Let $C$ be a closed convex subset of a real Hilbert space $H$. Let $\left\{x_{n}\right\}$ be a sequence in $H$ and $u \in H$. Let $q=P_{C} u$. If $\left\{x_{n}\right\}$ is such that $\omega_{w}\left(x_{n}\right) \subset C$ and satisfies the condition

$$
\left\|x_{n}-u\right\| \leq\|u-q\| \text { for all } n
$$

then $x_{n} \rightarrow q$.

\section{Main results}

In this section, we first introduce the following new iterative algorithm.

Algorithm 3.1. Let $C$ be a nonempty closed convex subset of a real Hilbert space $H, \varphi: C \rightarrow \mathbb{R}$ be a lower semicontinuous and convex real valued function, $\Theta: C \times C \rightarrow \mathbb{R}$ be an equilibrium bifunction and $T: C \rightarrow C$ be an asymptotically $k$-strict pseudo-contraction. Assume that $F(T) \cap \Omega$ is nonempty and bounded. Let $r$ be a positive parameter and $\delta \in(k, 1)$ be a constant. Let $\left\{\alpha_{n}\right\}$ be a sequence in $[0,1]$. Define the sequences $\left\{x_{n}\right\}$ and $\left\{y_{n}\right\}$ by the following manner:

$$
\left\{\begin{array}{l}
x_{0} \in C \text { chosen arbitrarily, } \\
\Theta\left(y_{n}, x\right)+\varphi(x)-\varphi\left(y_{n}\right)+\frac{1}{r}\left\langle K^{\prime}\left(y_{n}\right)-K^{\prime}\left(x_{n}\right), \eta\left(x, y_{n}\right)\right\rangle \geq 0, \forall x \in C, \\
z_{n}=\left(1-\alpha_{n}\right) x_{n}+\alpha_{n}\left[\delta y_{n}+(1-\delta) T^{n} y_{n}\right] \\
C_{n}=\left\{z \in C:\left\|z_{n}-z\right\|^{2} \leq\left\|x_{n}-z\right\|^{2}+\theta_{n}\right\} \\
Q_{n}=\left\{z \in C:\left\langle x_{n}-z, x_{0}-x_{n}\right\rangle \geq 0\right\} \\
x_{n+1}=P_{C},
\end{array}\right.
$$

where $\theta_{n}=\gamma_{n} \Delta_{n}^{2}, \Delta_{n}=\sup \left\{\left\|x_{n}-p\right\|: p \in F(T) \cap \Omega\right\}<\infty$.

Now we give a strong convergence result concerning iterative Algorithm 3.1 as follows.

Theorem 3.1. Let $C$ be a nonempty closed convex subset of a real Hilbert space $H$. Let $\varphi: C \rightarrow \mathbb{R}$ be a lower semicontinuous and convex functional. Let $\Theta: C \times C \rightarrow \mathbb{R}$ be an equilibrium bifunction satisfying conditions (H1)-(H3) and let $T: C \rightarrow C$ be an asymptotically $k$-strict pseudo-contraction. Assume that $F(T) \cap \Omega$ is nonempty and bounded. Assume that:

(i) $\eta: C \times C \rightarrow H$ is Lipschitz continuous with constant $\lambda>0$ such that; 
(a) $\eta(x, y)+\eta(y, x)=0, \quad \forall x, y \in C$,

(b) $\eta(\cdot, \cdot)$ is affine in the first variable,

(c) for each fixed $y \in C, x \mapsto \eta(y, x)$ is sequentially continuous from the weak topology to the weak topology;

(ii) $K: C \rightarrow \mathbb{R}$ is $\eta$-strongly convex with constant $\sigma>0$ and its derivative $K^{\prime}$ is not only sequentially continuous from the weak topology to the strong topology but also Lipschitz continuous with constant $\nu>0$ such that $\sigma \geq \lambda \nu$

(iii) for each $x \in C$; there exist a bounded subset $D_{x} \subset C$ and $z_{x} \in C$ such that, for any $C \ni y \notin D_{x}$,

$$
\Theta\left(y, z_{x}\right)+\varphi\left(z_{x}\right)-\varphi(y)+\frac{1}{r}\left\langle K^{\prime}(y)-K^{\prime}(x), \eta\left(z_{x}, y\right)\right\rangle<0 ;
$$

(iv) $\alpha_{n} \in[a, 1]$ for some $a \in(0,1)$.

Then the sequence $\left\{x_{n}\right\}$ generated iteratively by (3) converges strongly to $P_{F(T) \cap \Omega} x_{0}$ provided $S_{r}$ is firmly nonexpansive.

Proof. First, we show that the sequence $\left\{x_{n}\right\}$ is well-defined. It is obvious that $C_{n}$ and $Q_{n}$ are closed and convex. Let $p \in F(T) \cap \Omega$. From $y_{n}=S_{r} x_{n}$, we have

$$
\left\|y_{n}-p\right\|=\left\|S_{r} x_{n}-S_{r} p\right\| \leq\left\|x_{n}-p\right\| .
$$

From Lemma 2.2, (2) and (4), we obtain

(5)

$$
\begin{aligned}
\left\|z_{n}-p\right\|^{2} \leq & \left(1-\alpha_{n}\right)\left\|x_{n}-p\right\|^{2}+\alpha_{n}\left\|\delta\left(y_{n}-p\right)+(1-\delta)\left(T^{n} y_{n}-p\right)\right\|^{2} \\
= & \left(1-\alpha_{n}\right)\left\|x_{n}-p\right\|^{2}+\alpha_{n}\left[\delta\left\|y_{n}-p\right\|^{2}+(1-\delta)\left\|T^{n} y_{n}-p\right\|^{2}\right. \\
& \left.-\delta(1-\delta)\left\|T^{n} y_{n}-y_{n}\right\|^{2}\right] \\
\leq & \left(1-\alpha_{n}\right)\left\|x_{n}-p\right\|^{2}+\alpha_{n}\left\{\delta\left\|y_{n}-p\right\|^{2}+(1-\delta)\left[\left(1+\gamma_{n}\right)\left\|y_{n}-p\right\|^{2}\right.\right. \\
& \left.\left.+k\left\|y_{n}-T^{n} y_{n}\right\|^{2}\right]-\delta(1-\delta)\left\|y_{n}-T^{n} y_{n}\right\|^{2}\right\} \\
= & \left(1-\alpha_{n}\right)\left\|x_{n}-p\right\|^{2}+\alpha_{n}\left\{\left\|y_{n}-p\right\|^{2}+(1-\delta) \gamma_{n}\left\|y_{n}-p\right\|^{2}\right. \\
& \left.+(1-\delta)(k-\delta)\left\|y_{n}-T^{n} y_{n}\right\|^{2}\right\} \\
\leq & \left(1-\alpha_{n}\right)\left\|x_{n}-p\right\|^{2}+\alpha_{n}\left(1+\gamma_{n}\right)\left\|y_{n}-p\right\|^{2} \\
\leq & \left(1+\gamma_{n}\right)\left\|x_{n}-p\right\|^{2} .
\end{aligned}
$$

Hence, we have $\left\|z_{n}-p\right\|^{2} \leq\left\|x_{n}-p\right\|^{2}+\theta_{n}$. This implies that $p \in C_{n}$; thus,

$$
F(T) \cap \Omega \subset C_{n}
$$

for every $n \geq 0$. Next we show by induction that $F(T) \cap \Omega \subset C_{n} \cap Q_{n}$ for each $n \geq 0$. Since $F(T) \cap \Omega \subset C_{0}$ and $Q_{0}=C$, we get

$$
F(T) \cap \Omega \subset C_{0} \cap Q_{0} .
$$


Suppose that $F(T) \cap \Omega \subset C_{k} \cap Q_{k}$ for $k \in \mathbb{N}$. Then, there exists $x_{n+1} \in C_{k} \cap Q_{k}$ such that

$$
x_{n+1}=P_{C_{k} \cap Q_{k}} x_{0} .
$$

Therefore, for each $z \in C_{k} \cap Q_{k}$, we have

$$
\left\langle x_{k+1}-z, x_{0}-x_{k+1}\right\rangle \geq 0 .
$$

Note that $F(T) \cap \Omega \subset C_{k} \cap Q_{k}$. Hence, for any $z \in F(T) \cap \Omega$ we have

$$
\left\langle x_{k+1}-z, x_{0}-x_{k+1}\right\rangle \geq 0,
$$

therefore $z \in Q_{k+1}$. So, we get

$$
F(T) \cap \Omega \subset Q_{k+1} .
$$

From this and (6), we have

$$
F(T) \cap \Omega \subset C_{k+1} \cap Q_{k+1} .
$$

This denotes that the sequence $\left\{x_{n}\right\}$ is well-defined.

Since $F(T) \cap \Omega$ is a nonempty closed convex subset of $C$, there exists a unique $z^{\prime} \in F(T) \cap \Omega$ such that $z^{\prime}=P_{F(T) \cap \Omega} x_{0}$. From $x_{n+1}=P_{C_{n} \cap Q_{n}} x_{0}$, we have

$$
\left\|x_{n+1}-x_{0}\right\| \leq\left\|z-x_{0}\right\|
$$

for all $z \in C_{n} \cap Q_{n}$. Since $z^{\prime} \in F(T) \cap \Omega \subset C_{n} \cap Q_{n}$, we have

$$
\left\|x_{n+1}-x_{0}\right\| \leq\left\|z^{\prime}-x_{0}\right\|
$$

for every $n \geq 0$. Therefore, $\left\{x_{n}\right\}$ is bounded, so are $\left\{y_{n}\right\}$ and $\left\{z_{n}\right\}$. Since $x_{n}=P_{Q_{n}} x_{0}$ and $x_{n+1} \in Q_{n}$, we get

$$
\left\|x_{n}-x_{0}\right\| \leq\left\|x_{n+1}-x_{0}\right\| .
$$

This together with the boundedness of $\left\{\left\|x_{n}-x_{0}\right\|\right\}$ implies that $\lim _{n \rightarrow \infty}\left\|x_{n}-x_{0}\right\|$ exists. The fact that $x_{n+1} \in Q_{n}$ implies that $\left\langle x_{n+1}-x_{n}, x_{n}-x_{0}\right\rangle \geq 0$. Applying Lemma 2.2, we obtain

$$
\begin{aligned}
\left\|x_{n+1}-x_{n}\right\|^{2} & =\left\|\left(x_{n+1}-x_{0}\right)-\left(x_{n}-x_{0}\right)\right\|^{2} \\
& =\left\|x_{n+1}-x_{0}\right\|^{2}-\left\|x_{n}-x_{0}\right\|^{2}-2\left\langle x_{n+1}-x_{n}, x_{n}-x_{0}\right\rangle \\
& \leq\left\|x_{n+1}-x_{0}\right\|^{2}-\left\|x_{n}-x_{0}\right\|^{2} \\
& \rightarrow 0
\end{aligned}
$$

From $x_{n+1} \in C_{n}$, we have

$$
\begin{aligned}
\left\|x_{n}-z_{n}\right\| & \leq\left\|x_{n}-x_{n+1}\right\|+\left\|x_{n+1}-z_{n}\right\| \\
& \leq\left(2+\gamma_{n}\right)\left\|x_{n}-x_{n+1}\right\| \\
& \rightarrow 0
\end{aligned}
$$


For $p \in F(T) \cap \Omega$, noting that $S_{r}$ is firmly nonexpansive, we have

and hence,

$$
\begin{aligned}
\left\|y_{n}-p\right\|^{2} & =\left\|S_{r} x_{n}-S_{r} p\right\|^{2} \\
& \left.\leq \| S_{r} x_{n}-S_{r} p, x_{n}-p\right\rangle \\
& =\left\langle y_{n}-p, x_{n}-p\right\rangle \\
& =\frac{1}{2}\left\{\left\|y_{n}-p\right\|^{2}+\left\|x_{n}-p\right\|^{2}-\left\|x_{n}-y_{n}\right\|^{2}\right\},
\end{aligned}
$$

From (5), we have

$$
\left\|y_{n}-p\right\|^{2} \leq\left\|x_{n}-p\right\|^{2}-\left\|x_{n}-y_{n}\right\|^{2} .
$$

$$
\begin{aligned}
\left\|z_{n}-p\right\|^{2} & \leq\left(1-\alpha_{n}\right)\left\|x_{n}-p\right\|^{2}+\alpha_{n}\left(1+\gamma_{n}\right)\left\|y_{n}-p\right\|^{2} \\
& \leq\left(1-\alpha_{n}\right)\left\|x_{n}-p\right\|^{2}+\alpha_{n}\left(1+\gamma_{n}\right)\left\{\left\|x_{n}-p\right\|^{2}-\left\|x_{n}-y_{n}\right\|^{2}\right\} \\
& =\left(1+\alpha_{n} \gamma_{n}\right)\left\|x_{n}-p\right\|^{2}-\alpha_{n}\left(1+\gamma_{n}\right)\left\|x_{n}-y_{n}\right\|^{2},
\end{aligned}
$$

that is,

(9)

$$
\begin{aligned}
\left\|x_{n}-y_{n}\right\|^{2} & \leq \frac{1}{\alpha_{n}\left(1+\gamma_{n}\right)}\left\{\left\|x_{n}-p\right\|^{2}-\left\|z_{n}-p\right\|^{2}\right\}+\frac{\gamma_{n}\left\|x_{n}-p\right\|^{2}}{1+\gamma_{n}} \\
& \leq \frac{1}{\alpha_{n}\left(1+\gamma_{n}\right)}\left\|x_{n}-z_{n}\right\|\left\{\left\|x_{n}-p\right\|+\left\|z_{n}-p\right\|\right\}+\frac{\gamma_{n}\left\|x_{n}-p\right\|^{2}}{1+\gamma_{n}} \\
& \rightarrow 0 .
\end{aligned}
$$

Combining (8) and (9), we have

$$
\lim _{n \rightarrow \infty}\left\|y_{n+1}-y_{n}\right\|=0 \text {. }
$$

By the fact $\alpha_{n}(1-\delta)\left(T^{n} y_{n}-y_{n}\right)=z_{n}-\left(1-\alpha_{n}\right) x_{n}-\alpha_{n} y_{n}$, we get

$$
\left\|\alpha_{n}(1-\delta)\left(T^{n} y_{n}-y_{n}\right)\right\| \leq\left\|z_{n}-x_{n}\right\|+\alpha_{n}\left\|x_{n}-y_{n}\right\| \rightarrow 0,
$$

which implies that

$$
\left\|T^{n} y_{n}-y_{n}\right\| \rightarrow 0
$$

Next we show that

$$
\lim _{n \rightarrow \infty}\left\|y_{n}-T y_{n}\right\|=0 .
$$

As a matter of fact, we have

$$
\begin{aligned}
\left\|y_{n}-T y_{n}\right\| & \leq\left\|y_{n}-T^{n} y_{n}\right\|+\left\|T^{n} y_{n}-T^{n+1} y_{n}\right\|+\left\|T^{n+1} y_{n}-T y_{n}\right\| \\
& \leq\left(1+L_{1}\right)\left\|y_{n}-T^{n} y_{n}\right\|+\left\|T^{n} y_{n}-T^{n+1} y_{n}\right\| .
\end{aligned}
$$

Note that

$$
\begin{aligned}
\left\|T^{n} y_{n}-T^{n+1} y_{n}\right\| \leq & \left\|T^{n} y_{n}-y_{n}\right\|+\left\|y_{n}-y_{n+1}\right\|+\left\|y_{n+1}-T^{n+1} y_{n+1}\right\| \\
& +\left\|T^{n+1} y_{n+1}-T^{n+1} y_{n}\right\| \\
\leq & \left\|T^{n} y_{n}-y_{n}\right\|+\left(1+L_{n+1}\right)\left\|y_{n}-y_{n+1}\right\| \\
& +\left\|y_{n+1}-T^{n+1} y_{n+1}\right\| .
\end{aligned}
$$


Therefore, we have

$$
\begin{aligned}
\left\|y_{n}-T y_{n}\right\| \leq & \left(2+L_{1}\right)\left\|y_{n}-T^{n} y_{n}\right\|+\left(1+L_{n+1}\right)\left\|y_{n}-y_{n+1}\right\| \\
& +\left\|y_{n+1}-T^{n+1} y_{n+1}\right\| \rightarrow 0
\end{aligned}
$$

Since $\left\{y_{n}\right\}$ is bounded, there exists a subsequence $\left\{y_{n_{i}}\right\}$ of $\left\{y_{n}\right\}$ which converges weakly to $w$. From (7), we also obtain that $T y_{n_{i}} \rightarrow w$ weakly. Next we show that $w \in \Omega$. Since $y_{n}=S_{r} x_{n}$, we derive

$$
\Theta\left(y_{n}, x\right)+\varphi(x)-\varphi\left(y_{n}\right)+\frac{1}{r}\left\langle K^{\prime}\left(y_{n}\right)-K^{\prime}\left(x_{n}\right), \eta\left(x, y_{n}\right)\right\rangle \geq 0, \quad \forall x \in C .
$$

From the monotonicity of $\Theta$, we have

$$
\frac{1}{r}\left\langle K^{\prime}\left(y_{n}\right)-K^{\prime}\left(x_{n}\right), \eta\left(x, y_{n}\right)\right\rangle+\varphi(x)-\varphi\left(y_{n}\right) \geq-\Theta\left(y_{n}, x\right) \geq \Theta\left(x, y_{n}\right),
$$

and hence

$$
\left\langle\frac{K^{\prime}\left(y_{n_{i}}\right)-K^{\prime}\left(x_{n_{i}}\right)}{r}, \eta\left(x, y_{n_{i}}\right)\right\rangle+\varphi(x)-\varphi\left(y_{n_{i}}\right) \geq \Theta\left(x, y_{n_{i}}\right) .
$$

Since $\frac{K^{\prime}\left(y_{n_{i}}\right)-K^{\prime}\left(x_{n_{i}}\right)}{r} \rightarrow 0$ and $y_{n_{i}} \rightarrow w$ weakly, from the weak lower semicontinuity of $\varphi$ and $\Theta(x, y)$ in the second variable $y$, we have

$$
\Theta(x, w)+\varphi(w)-\varphi(x) \leq 0
$$

for all $x \in C$. For $0<t \leq 1$ and $x \in C$, let $x_{t}=t x+(1-t) w$. Since $x \in C$ and $w \in C$, we have $x_{t} \in C$ and hence $\Theta\left(x_{t}, w\right)+\varphi(w)-\varphi\left(x_{t}\right) \leq 0$. From the convexity of equilibrium bifunction $\Theta(x, y)$ in the second variable $y$, we have

$$
\begin{aligned}
0 & =\Theta\left(x_{t}, x_{t}\right)+\varphi\left(x_{t}\right)-\varphi\left(x_{t}\right) \\
& \leq t \Theta\left(x_{t}, x\right)+(1-t) \Theta\left(x_{t}, w\right)+t \varphi(x)+(1-t) \varphi(w)-\varphi\left(x_{t}\right) \\
& \leq t\left[\Theta\left(x_{t}, x\right)+\varphi(x)-\varphi\left(x_{t}\right)\right],
\end{aligned}
$$

and hence $\Theta\left(x_{t}, x\right)+\varphi(x)-\varphi\left(x_{t}\right) \geq 0$. Then, we have

$$
\Theta(w, x)+\varphi(x)-\varphi(w) \geq 0
$$

for all $x \in C$ and hence $w \in \Omega$.

We shall prove that $w \in F(T)$. As a matter of fact, Lemma 2.3 and (10) guarantee that every weak limit point of $\left\{y_{n}\right\}$ is a fixed point of $T$. That is, $\omega_{w}\left(y_{n}\right) \subset F(T)$. Hence, $w \in F(T)$.

Therefore, we have

$$
w \in F(T) \cap \Omega .
$$

This fact, the inequality (7) and Lemma 2.4 ensure the strong convergence of $\left\{x_{n}\right\}$ to $P_{F(T) \cap \Omega} x_{0}$. This completes the proof.

As a direct consequence of Theorem 3.1, we obtain the following. 
Corollary 3.1. Let $C$ be a nonempty closed convex subset of a real Hilbert space $H$. Let $\varphi: C \rightarrow \mathbb{R}$ be a lower semicontinuous and convex functional. Let $\Theta: C \times C \rightarrow \mathbb{R}$ be an equilibrium bifunction satisfying conditions (H1)(H3) and let $T: C \rightarrow C$ be an asymptotically nonexpansive mapping such that $F(T) \cap \Omega \neq \emptyset$. Assume that:

(i) $\eta: C \times C \rightarrow H$ is Lipschitz continuous with constant $\lambda>0$ such that;

(a) $\eta(x, y)+\eta(y, x)=0, \forall x, y \in C$,

(b) $\eta(\cdot, \cdot)$ is affine in the first variable,

(c) for each fixed $y \in C, x \mapsto \eta(y, x)$ is sequentially continuous from the weak topology to the weak topology;

(ii) $K: C \rightarrow \mathbb{R}$ is $\eta$-strongly convex with constant $\sigma>0$ and its derivative $K^{\prime}$ is not only sequentially continuous from the weak topology to the strong topology but also Lipschitz continuous with constant $\nu>0$ such that $\sigma \geq \lambda \nu$

(iii) for each $x \in C$; there exist a bounded subset $D_{x} \subset C$ and $z_{x} \in C$ such that, for any $C \ni y \notin D_{x}$,

$$
\Theta\left(y, z_{x}\right)+\varphi\left(z_{x}\right)-\varphi(y)+\frac{1}{r}\left\langle K^{\prime}(y)-K^{\prime}(x), \eta\left(z_{x}, y\right)\right\rangle<0 ;
$$

(iv) $\alpha_{n} \in[a, 1]$ for some $a \in(0,1)$.

Then the sequence $\left\{x_{n}\right\}$ generated iteratively by (3) converges strongly to $P_{F(T) \cap \Omega} x_{0}$ provided $S_{r}$ is firmly nonexpansive.

Corollary 3.2. Let $C$ be a nonempty closed convex subset of a real Hilbert space $H$. Let $T: C \rightarrow C$ be an asymptotically $k$-strict pseudo-contraction such that $F(T)$ is nonempty and bounded. Let $\delta \in(k, 1)$ be a constant and $\left\{\alpha_{n}\right\}$ be a sequence in $[0,1]$. Assume $\alpha_{n} \in[a, 1]$ for some $a \in(0,1)$. For given $x_{0} \in C$ arbitrarily, let the sequence $\left\{x_{n}\right\}$ generated iteratively by

$$
\left\{\begin{array}{l}
z_{n}=\left(1-\alpha_{n}\right) x_{n}+\alpha_{n}\left[\delta x_{n}+(1-\delta) T^{n} x_{n}\right] \\
C_{n}=\left\{z \in C:\left\|z_{n}-z\right\|^{2} \leq\left\|x_{n}-z\right\|^{2}+\theta_{n}\right\} \\
Q_{n}=\left\{z \in C:\left\langle x_{n}-z, x_{0}-x_{n}\right\rangle \geq 0\right\} \\
x_{n+1}=P_{C_{n} \cap Q_{n}} x_{0},
\end{array}\right.
$$

where $\theta_{n}=\gamma_{n} \Delta_{n}^{2}, \Delta_{n}=\sup \left\{\left\|x_{n}-p\right\|: p \in F(T)\right\}<\infty$. Then the sequence $\left\{x_{n}\right\}$ defined by (11) converges strongly to $P_{F(T)} x_{0}$.

Proof. Set $\varphi(x)=0$ and $\Theta(x, y)=0$ for all $x, y \in C$ and put $r=1$. Take $K(x)=\frac{\|x\|^{2}}{2}$ and $\eta(y, x)=y-x$ for all $x, y \in C$. Then we have $y_{n}=x_{n}$. Hence, by the similar argument as that in the proof of Theorem 3.1, we can obtain the desired result. This completes the proof.

Corollary 3.3. Let $C$ be a nonempty closed convex subset of a real Hilbert space $H$. Let $T: C \rightarrow C$ be an asymptotically nonexpansive mappings such that $F(T)$ is nonempty and bounded. Let $\delta \in(k, 1)$ be a constant and $\left\{\alpha_{n}\right\}$ be 
a sequence in $[0,1]$. Assume $\alpha_{n} \in[a, 1]$ for some $a \in(0,1)$. Then the sequence $\left\{x_{n}\right\}$ defined by $(11)$ converges strongly to $P_{F(T)} x_{0}$.

Now we give another iterative algorithm as follows.

Algorithm 3.2. Let $C$ be a nonempty closed convex subset of a real Hilbert space $H, \varphi: C \rightarrow \mathbb{R}$ be a lower semicontinuous and convex real valued function, $\Theta: C \times C \rightarrow \mathbb{R}$ be an equilibrium bifunction and $T: C \rightarrow C$ be an asymptotically $k$-strict pseudo-contraction. Let $r$ be a positive parameter and $\delta \in(k, 1)$ be a constant. Let $\left\{\alpha_{n}\right\}$ be a sequence in $[0,1]$. Define the sequences $\left\{x_{n}\right\}$ and $\left\{y_{n}\right\}$ by the following manner:

$$
\left\{\begin{array}{l}
x_{0} \in C \text { chosen arbitrarily, } \\
\Theta\left(y_{n}, x\right)+\varphi(x)-\varphi\left(y_{n}\right)+\frac{1}{r}\left\langle K^{\prime}\left(y_{n}\right)-K^{\prime}\left(x_{n}\right), \eta\left(x, y_{n}\right)\right\rangle \geq 0, \forall x \in C, \\
x_{n+1}=\left(1-\alpha_{n}\right) x_{n}+\alpha_{n}\left[\delta y_{n}+(1-\delta) T^{n} y_{n}\right] .
\end{array}\right.
$$

Finally we state and prove a weak convergence theorem concerning Algorithm 3.2 .

Theorem 3.2. Let $C$ be a nonempty closed convex subset of a real Hilbert space $H$. Let $\varphi: C \rightarrow \mathbb{R}$ be a lower semicontinuous and convex functional. Let $\Theta: C \times C \rightarrow \mathbb{R}$ be an equilibrium bifunction satisfying conditions (H1)-(H3) and let $T: C \rightarrow C$ be an asymptotically $k$-strict pseudo-contraction such that $F(T) \cap \Omega \neq \emptyset$. Assume that:

(i) $\eta: C \times C \rightarrow H$ is Lipschitz continuous with constant $\lambda>0$ such that;

(a) $\eta(x, y)+\eta(y, x)=0, \quad \forall x, y \in C$,

(b) $\eta(\cdot, \cdot)$ is affine in the first variable,

(c) for each fixed $y \in C, x \mapsto \eta(y, x)$ is sequentially continuous from the weak topology to the weak topology;

(ii) $K: C \rightarrow \mathbb{R}$ is $\eta$-strongly convex with constant $\sigma>0$ and its derivative $K^{\prime}$ is not only sequentially continuous from the weak topology to the strong topology but also Lipschitz continuous with constant $\nu>0$ such that $\sigma \geq \lambda \nu$

(iii) for each $x \in C$, there exist a bounded subset $D_{x} \subset C$ and $z_{x} \in C$ such that, for any $C \ni y \notin D_{x}$,

$$
\Theta\left(y, z_{x}\right)+\varphi\left(z_{x}\right)-\varphi(y)+\frac{1}{r}\left\langle K^{\prime}(y)-K^{\prime}(x), \eta\left(z_{x}, y\right)\right\rangle<0
$$

(iv) $\alpha_{n} \in[a, b]$ for some $a, b \in(0,1)$ and $\sum_{n=1}^{\infty} \gamma_{n}<\infty$.

Then the sequence $\left\{x_{n}\right\}$ generated iteratively by (12) converges weakly to $w \in$ $P_{F(T) \cap \Omega}$ provided $S_{r}$ is firmly nonexpansive, where $w=\lim _{n \rightarrow \infty} P_{F(T) \cap \Omega}\left(x_{n}\right)$.

Proof. By Lemma 2.1, $\left\{x_{n}\right\}$ and $\left\{y_{n}\right\}$ are all well-defined. Let $p \in F(T) \cap \Omega$, from $y_{n}=S_{r} x_{n}$, we have

$$
\left\|y_{n}-p\right\|=\left\|S_{r} x_{n}-S_{r} p\right\| \leq\left\|x_{n}-p\right\| .
$$


Therefore, we have

$$
\begin{aligned}
\left\|x_{n+1}-p\right\|^{2} \leq & \left(1-\alpha_{n}\right)\left\|x_{n}-p\right\|^{2}+\alpha_{n}\left\|\delta\left(y_{n}-p\right)+(1-\delta)\left(T^{n} y_{n}-p\right)\right\|^{2} \\
= & \left(1-\alpha_{n}\right)\left\|x_{n}-p\right\|^{2}+\alpha_{n}\left[\delta\left\|y_{n}-p\right\|^{2}+(1-\delta)\left\|T^{n} y_{n}-p\right\|^{2}\right. \\
& \left.-\delta(1-\delta)\left\|y_{n}-T^{n} y_{n}\right\|^{2}\right] \\
\leq & \left(1-\alpha_{n}\right)\left\|x_{n}-p\right\|^{2}+\alpha_{n}\left\{\delta\left\|y_{n}-p\right\|^{2}+(1-\delta)\left[\left(1+\gamma_{n}\right)\right.\right. \\
& \left.\left.\times\left\|y_{n}-p\right\|^{2}+k\left\|y_{n}-T^{n} y_{n}\right\|^{2}\right]-\delta(1-\delta)\left\|y_{n}-T^{n} y_{n}\right\|^{2}\right\} \\
= & \left(1-\alpha_{n}\right)\left\|x_{n}-p\right\|^{2}+\alpha_{n}\left\{\left[1+(1-\delta) \gamma_{n}\right]\left\|y_{n}-p\right\|^{2}\right. \\
& \left.+(1-\delta)(k-\delta)\left\|y_{n}-T y_{n}\right\|^{2}\right\} \\
\leq & \left(1-\alpha_{n}\right)\left\|x_{n}-p\right\|^{2}+\alpha_{n}\left(1+\gamma_{n}\right)\left\|y_{n}-p\right\|^{2} \\
\leq & \left(1+\gamma_{n}\right)\left\|x_{n}-p\right\|^{2} .
\end{aligned}
$$

This implies that $\lim _{n \rightarrow \infty}\left\|x_{n}-p\right\|$ exists. Hence, $\left\{x_{n}\right\},\left\{y_{n}\right\}$ are all bounded and

$$
\lim _{n \rightarrow \infty}\left\|x_{n+1}-x_{n}\right\|=0 .
$$

Next, for $p \in F(T) \cap \Omega$, noting $S_{r}$ is firmly nonexpansive, we have

$$
\begin{aligned}
\left\|y_{n}-p\right\|^{2} & =\left\|S_{r} x_{n}-S_{r} p\right\|^{2} \\
& \left.\leq \| S_{r} x_{n}-S_{r} p, x_{n}-p\right\rangle \\
& =\left\langle y_{n}-p, x_{n}-p\right\rangle \\
& =\frac{1}{2}\left\{\left\|y_{n}-p\right\|^{2}+\left\|x_{n}-p\right\|^{2}-\left\|x_{n}-y_{n}\right\|^{2}\right\},
\end{aligned}
$$

and hence,

$$
\left\|y_{n}-p\right\|^{2} \leq\left\|x_{n}-p\right\|^{2}-\left\|x_{n}-y_{n}\right\|^{2} .
$$

Therefore, we have

$$
\begin{aligned}
\left\|x_{n+1}-p\right\|^{2} \leq & \left(1-\alpha_{n}\right)\left\|x_{n}-p\right\|^{2}+\alpha_{n}\left(1+\gamma_{n}\right)\left\|y_{n}-p\right\|^{2} \\
\leq & \left(1-\alpha_{n}\right)\left\|x_{n}-p\right\|^{2}+\alpha_{n}\left(\left\|x_{n}-p\right\|^{2}-\left\|x_{n}-y_{n}\right\|^{2}\right) \\
& +\alpha_{n} \gamma_{n}\left\|y_{n}-p\right\|^{2} \\
\leq & \left\|x_{n}-p\right\|^{2}-\alpha_{n}\left\|x_{n}-y_{n}\right\|^{2}+\alpha_{n} \gamma_{n}\left\|y_{n}-p\right\|^{2} .
\end{aligned}
$$

So, we obtain

$$
\left\|x_{n}-y_{n}\right\|^{2} \leq \frac{1}{\alpha_{n}}\left\{\left\|x_{n}-p\right\|^{2}-\left\|x_{n+1}-p\right\|^{2}\right\}+\gamma_{n}\left\|y_{n}-p\right\|^{2} \rightarrow 0 .
$$

As $\left\{x_{n}\right\}$ is bounded, there exists a subsequence $\left\{x_{n_{i}}\right\}$ of $\left\{x_{n}\right\}$ which converges weakly to $w$. From $\left\|x_{n}-y_{n}\right\| \rightarrow 0$, we also have that $y_{n_{i}} \rightarrow w$ weakly. First, as in the proof of Theorem 3.1, we can show $w \in \Omega$. Let us show that $w \in F(T)$. 
Let $p \in F(T) \cap \Omega$. Since $\alpha_{n}(1-\delta) T^{n} y_{n}=x_{n+1}-\left(1-\alpha_{n}\right) x_{n}-\alpha \delta y_{n}$, we have

$$
\begin{aligned}
\left\|\alpha_{n}(1-\delta)\left(T^{n} y_{n}-x_{n}\right)\right\| & =\left\|x_{n+1}-x_{n}+\alpha_{n} \delta\left(x_{n}-y_{n}\right)\right\| \\
& \leq\left\|x_{n+1}-x_{n}\right\|+\alpha_{n}\left\|x_{n}-y_{n}\right\| \\
& \rightarrow 0
\end{aligned}
$$

which implies that

$$
\left\|T^{n} y_{n}-x_{n}\right\| \rightarrow 0
$$

Hence

$$
\left\|T^{n} y_{n}-y_{n}\right\| \leq\left\|T^{n} y_{n}-x_{n}\right\|+\left\|y_{n}-x_{n}\right\| \rightarrow 0 .
$$

Repeating the similar argument as Theorem 3.1, we can obtain

$$
\lim _{n \rightarrow \infty}\left\|y_{n}-T y_{n}\right\|=0 \text {. }
$$

From this and $y_{n_{i}} \rightarrow w$ weakly, we obtain $w \in F(T)$. Then, $w \in F(T) \cap \Omega$.

Let $\left\{x_{n_{j}}\right\}$ be another subsequence of $\left\{x_{n}\right\}$ such that $x_{n_{j}} \rightarrow w^{\prime}$ weakly. Then, we have

$$
w^{\prime} \in F(T) \cap \Omega \text {. }
$$

If $w \neq w^{\prime}$, from the opial theorem [20], we get

$$
\begin{aligned}
\lim _{n \rightarrow \infty}\left\|x_{n}-w\right\| & =\liminf _{i \rightarrow \infty}\left\|x_{n_{i}}-w\right\| \\
& <\liminf _{i \rightarrow \infty}\left\|x_{n_{i}}-w^{\prime}\right\| \\
& =\lim _{n \rightarrow \infty}\left\|x_{n}-w^{\prime}\right\| \\
& =\liminf _{j \rightarrow \infty}\left\|x_{n_{j}}-w^{\prime}\right\| \\
& <\liminf _{j \rightarrow \infty}\left\|x_{n_{j}}-w\right\| \\
& =\lim _{n \rightarrow \infty}\left\|x_{n}-w\right\| .
\end{aligned}
$$

This is a contradiction. So we have $w=w^{\prime}$. This implies that

$$
x_{n} \rightarrow F(T) \cap \Omega \quad \text { weakly. }
$$

Let $z_{n}=P_{F(T) \cap \Omega}\left(x_{n}\right)$. Since $w \in F(T) \cap \Omega$, we have $\left\langle x_{n}-z_{n}, z_{n}-w\right\rangle \geq 0$. Hence, we have that $\left\{z_{n}\right\}$ converges strongly to some $w_{0} \in F(T) \cap \Omega$. Since $\left\{x_{n}\right\}$ converges weakly to $w$, we have

$$
\left\langle w-w_{0}, w_{0}-w\right\rangle \geq 0 .
$$

Therefore, we obtain

$$
w=w_{0}=\lim _{n \rightarrow \infty} P_{F(T) \cap \Omega}\left(x_{n}\right) .
$$

This completes the proof. 
Corollary 3.4. Let $C$ be a nonempty closed convex subset of a real Hilbert space $H$. Let $\varphi: C \rightarrow \mathbb{R}$ be a lower semicontinuous and convex functional. Let $\Theta: C \times C \rightarrow \mathbb{R}$ be an equilibrium bifunction satisfying conditions (H1)(H3) and let $T: C \rightarrow C$ be an asymptotically nonexpansive mapping such that $F(T) \cap \Omega \neq \emptyset$. Assume that:

(i) $\eta: C \times C \rightarrow H$ is Lipschitz continuous with constant $\lambda>0$ such that; (a) $\eta(x, y)+\eta(y, x)=0, \forall x, y \in C$,

(b) $\eta(\cdot, \cdot)$ is affine in the first variable,

(c) for each fixed $y \in C, x \mapsto \eta(y, x)$ is sequentially continuous from the weak topology to the weak topology;

(ii) $K: C \rightarrow \mathbb{R}$ is $\eta$-strongly convex with constant $\sigma>0$ and its derivative $K^{\prime}$ is not only sequentially continuous from the weak topology to the strong topology but also Lipschitz continuous with constant $\nu>0$ such that $\sigma \geq \lambda \nu$

(iii) for each $x \in C$, there exist a bounded subset $D_{x} \subset C$ and $z_{x} \in C$ such that, for any $C \ni y \notin D_{x}$,

$$
\Theta\left(y, z_{x}\right)+\varphi\left(z_{x}\right)-\varphi(y)+\frac{1}{r}\left\langle K^{\prime}(y)-K^{\prime}(x), \eta\left(z_{x}, y\right)\right\rangle<0
$$

(iv) $\alpha_{n} \in[a, b]$ for some $a, b \in(0,1)$ and $\sum_{n=0}^{\infty} \gamma_{n}<\infty$.

Then the sequence $\left\{x_{n}\right\}$ generated iteratively by (12) converges weakly to $w \in$ $P_{F(T) \cap \Omega}$ provided $S_{r}$ is firmly nonexpansive, where $w=\lim _{n \rightarrow \infty} P_{F(T) \cap \Omega}\left(x_{n}\right)$.

Corollary 3.5. Let $C$ be a nonempty closed convex subset of a real Hilbert space $H$. Let $\varphi: C \rightarrow \mathbb{R}$ be a lower semicontinuous and convex functional. Let $\Theta: C \times C \rightarrow \mathbb{R}$ be an equilibrium bifunction satisfying conditions (H1)-(H3) such that $\Omega \neq \emptyset$. Assume that:

(i) $\eta: C \times C \rightarrow H$ is Lipschitz continuous with constant $\lambda>0$ such that; (a) $\eta(x, y)+\eta(y, x)=0, \forall x, y \in C$,

(b) $\eta(\cdot, \cdot)$ is affine in the first variable,

(c) for each fixed $y \in C, x \mapsto \eta(y, x)$ is sequentially continuous from the weak topology to the weak topology;

(ii) $K: C \rightarrow \mathbb{R}$ is $\eta$-strongly convex with constant $\sigma>0$ and its derivative $K^{\prime}$ is not only sequentially continuous from the weak topology to the strong topology but also Lipschitz continuous with constant $\nu>0$ such that $\sigma \geq \lambda \nu$

(iii) for each $x \in C$, there exist a bounded subset $D_{x} \subset C$ and $z_{x} \in C$ such that, for any $C \ni y \notin D_{x}$,

$$
\Theta\left(y, z_{x}\right)+\varphi\left(z_{x}\right)-\varphi(y)+\frac{1}{r}\left\langle K^{\prime}(y)-K^{\prime}(x), \eta\left(z_{x}, y\right)\right\rangle<0
$$


Let the sequences $\left\{x_{n}\right\}$ and $\left\{y_{n}\right\}$ be generated by

$$
\left\{\begin{array}{l}
x_{0} \in C \text { chosen arbitrarily, } \\
\Theta\left(y_{n}, x\right)+\varphi(x)-\varphi\left(y_{n}\right)+\frac{1}{r}\left\langle K^{\prime}\left(y_{n}\right)-K^{\prime}\left(x_{n}\right), \eta\left(x, y_{n}\right)\right\rangle \geq 0, \forall x \in C, \\
x_{n+1}=\left(1-\alpha_{n}\right) x_{n}+\alpha_{n} y_{n},
\end{array}\right.
$$

where $r$ is a positive parameter and $\left\{\alpha_{n}\right\}$ is a sequence $\in[a, b]$ for some $a, b \in$ $(0,1)$.

Then the sequence $\left\{x_{n}\right\}$ converges weakly to $w \in \Omega$ provided $S_{r}$ is firmly nonexpansive, where $w=\lim _{n \rightarrow \infty} P_{\Omega}\left(x_{n}\right)$.

Proof. Taking $T=I$ in Theorem 3.2, we can obtain our desired result. This completes the proof.

Corollary 3.6. Let $C$ be a nonempty closed convex subset of a real Hilbert space $H$. Let $T: C \rightarrow C$ be an asymptotically $k$-strict pseudo-contraction such that $F(T) \neq \emptyset$. Let $\delta \in(k, 1)$ be a constant and $\left\{\alpha_{n}\right\}$ be a real sequence in $[0,1]$. Assume that $\alpha_{n} \in[a, b]$ for some $a, b \in(0,1)$ and $\sum_{n=1}^{\infty} \gamma_{n}<\infty$. For given $x_{0} \in C$ arbitrarily, let the sequence $\left\{x_{n}\right\}$ generated iteratively by

$$
x_{n+1}=\left(1-\alpha_{n}\right) x_{n}+\alpha_{n}\left[\delta y_{n}+(1-\delta) T^{n} y_{n}\right] .
$$

Then $\left\{x_{n}\right\}$ converges weakly to $w \in P_{F(T)}$, where $w=\lim _{n \rightarrow \infty} P_{F(T)}\left(x_{n}\right)$.

\section{References}

[1] E. Blum and W. Oettli, From optimization and variational inequalities to equilibrium problems, Math. Student 63 (1994), no. 1-4, 123-145.

[2] O. Chadli, I. V. Konnov, and J. C. Yao, Descent methods for equilibrium problems in a Banach space, Comput. Math. Appl. 48 (2004), no. 3-4, 609-616.

[3] O. Chadli, S. Schaible, and J. C. Yao, Regularized equilibrium problems with application to noncoercive hemivariational inequalities, J. Optim. Theory Appl. 121 (2004), no. 3, $571-596$.

[4] O. Chadli, N. C. Wong, and J. C. Yao, Equilibrium problems with applications to eigenvalue problems, J. Optim. Theory Appl. 117 (2003), no. 2, 245-266.

[5] P. L. Combettes and S. A. Hirstoaga, Equilibrium programming in Hilbert spaces, J. Nonlinear Convex Anal. 6 (2005), no. 1, 117-136.

[6] X. P. Ding, Y. C. Lin, and J. C. Yao, Predictor-corrector algorithms for solving generalized mixed implicit quasi-equilibrium problems, Appl. Math. Mech. (English Ed.) 27 (2006), no. 9, 1157-1164.

[7] S. D. Flåm and A. S. Antipin, Equilibrium programming using proximal-like algorithms, Math. Programming 78 (1997), no. 1, Ser. A, 29-41.

[8] T. H. Kim and H. K. Xu, Strong convergence of modified Mann iterations for asymptotically nonexpansive mappings and semigroups, Nonlinear Anal. 64 (2006), no. 5, $1140-1152$.

[9] - Convergence of the modified Mann's iteration method for asymptotically strict pseudo-contractions, Nonlinear Analysis: Theory, Methods \& Applications 68 (2008), no. 9, 2828-2836.

[10] I. V. Konnov, S. Schaible, and J. C. Yao, Combined relaxation method for mixed equilibrium problems, J. Optim. Theory Appl. 126 (2005), no. 2, 309-322. 
[11] C. Matinez-Yanes and H. K. Xu, Strong convergence of the CQ method for fixed point iteration processes, Nonlinear Anal. 64 (2006), no. 11, 2400-2411.

[12] M. A. Noor, Fundamentals of equilibrium problems, Math. Inequal. Appl. 9 (2006), no. 3, 529-566.

[13] Z. Opial, Weak convergence of the sequence of successive approximations for nonexpansive mappings, Bull. Amer. Math. Soc. 73 (1967), 591-597.

[14] S. Plubtieng and R. Punpaeng, A general iterative method for equilibrium problems and fixed point problems in Hilbert spaces, J. Math. Anal. Appl. 336 (2007), no. 1, 455-469.

[15] A. Tada and W. Takahashi, Strong convergence theorem for an equilibrium problem and a nonexpansive mapping, Nonlinear analysis and convex analysis, 609-617, Yokohama Publ., Yokohama, 2007.

[16] _ Weak and strong convergence theorems for a nonexpansive mapping and an equilibrium problem, J. Optim. Theory Appl. 133 (2007), no. 3, 359-370.

[17] S. Takahashi and W. Takahashi, Viscosity approximation methods for equilibrium problems and fixed point problems in Hilbert spaces, J. Math. Anal. Appl. 331 (2007), no. $1,506-515$.

[18] Y. Yao, Y. C. Liou, and J. C. Yao, Convergence theorem for equilibrium problems and fixed point problems of infinite family of nonexpansive mappings, Fixed Point Theory Appl. 2007, Art. ID 64363, 12 pp.

[19] _ A new hybrid iterative algorithm for fixed point problems, variational inequality problems and mixed equilibrium problems, Fixed Point Theory and Applications 2008 (2008), Article ID 417089, 15 pages.

[20] Y. Yao, M. A. Noor, and Y. C. Liou, On iterative methods for equilibrium problems, Nonlinear Anal. 70 (2009), no. 1, 497-509.

[21] L. C. Zeng and J. C. Yao, A hybrid iterative scheme for mixed equilibrium problems and fixed point problems , J. Comput. Appl. Math. 214 (2008), no. 1, 186-201.

[22] L. C. Zeng, S. Y. Wu, and J. C. Yao, Generalized KKM theorem with applications to generalized minimax inequalities and generalized equilibrium problems, Taiwanese J. Math. 10 (2006), no. 6, 1497-1514.

YONGHONG YAO

Department of Mathematics

Tianjin Polytechnic University

Tianjin 300160, ChinA

E-mail address: yaoyonghong@yahoo.cn

HAIYUN ZHOU

Department of Mathematics

Shijiazhuang Mechanical Engineering College

SHIJIAZHUANG 050003, ChinA

E-mail address: witman66@yahoo.com.cn

YEONG-CHENG LIOU

Department of Information Management

Cheng Shiu University

KAOHSIUNG 833, TAIWAN

E-mail address: simplex_liou@hotmail.com 